Proceedings of the 48th International School and Conference on the Physics of Semiconductors "Jaszowiec 2019"

\title{
Investigation of the Spectrum of Exciton Excited States in Self-Organized InAs/AlGaAs Quantum Dots
}

\author{
A.I. Galimov*, M.V. Rakhlin, K.G. Belyaev, G.V. Klimko, \\ E.A. Evropeytsev and A.A. TOROpov \\ Ioffe Institute, 26 Polytechnicheskaya Str., 194021 St. Petersburg, Russia
}

\begin{abstract}
We performed comparative optical spectroscopic studies of the excitonic spectrum in self-organized InAs $/ \mathrm{Al}_{0.3} \mathrm{Ga}_{0.7} \mathrm{As}$ and InAs/GaAs quantum dots. The exciton excited states were revealed by either measuring photoluminescence spectra using different excitation pump powers or by carrying out selective resonant excitation of the quantum dot photoluminescence through the excited states. As a result, we evaluated average energies of the excitonic transitions and elucidated dominant mechanisms of exciton relaxation from the excited states to the ground one for both types of quantum dots.
\end{abstract}

DOI: 10.12693/APhysPolA.136.613

PACS/topics: 73.21.La, 71.35.--y, 78.55.Cr, 72.10.Di

\section{Introduction}

Self-organized quantum dots (QDs) grown by epitaxial methods are considered as a basis for various applications in quantum photonics, because of their unique properties such as a small emission linewidth, fast radiative decay time, and high quantum efficiency [1]. Among such applications is generation of single photons with high degree of indistinguishability, required for implementation of linear optics schemes of quantum computing [2]. Most of the currently used QD systems (e.g. $\mathrm{CdSe} / \mathrm{Zn}(\mathrm{S}) \mathrm{Se}, \mathrm{InP} /(\mathrm{Al}, \mathrm{Ga}, \mathrm{In}) \mathrm{P}, \mathrm{CdTe} / \mathrm{ZnTe})$ operate in a relatively narrow wavelength range. The exception is the (In, Al, Ga) As material system, which allows one to design single photon sources in the wide infrared (IR) spectral range between $0.80 \mathrm{eV}$ (InAs/InGaAs) and $1.38 \mathrm{eV}$ (InGaAs/GaAs). More recently, the authors of Ref. [3] have expanded the operational spectral range to the red visible light (up to $1.96 \mathrm{eV}$ ) by means of InAs/AlGaAs QDs. The shift in emission toward higher energies in comparison with InAs/GaAs QD arises from a combination of the large barrier band gap and also from possible interdiffusion of $\mathrm{Al}$ into the QDs, leading to an additional increase of the band gap of the QD material [4]. The InAs/AlGaAs QDs operating spectral range is attractive since it corresponds to the maximum sensitivity of modern single-photon $\mathrm{Si}$ avalanche photodiodes. However, the highest degree of indistinguishability of single photons yet achieved in these structures with non-resonant over-barrier optical pumping was only about $30 \%$ [5]. In order to improve the photon indistinguishability, one primarily needs to realize either coherent resonant pumping of the ground exciton $s$-shell [6] or quasi-resonant [7] pumping of exciton excited states (e.g. through $p$-shell) that results in significant reduction

\footnotetext{
* corresponding author
}

of spectral diffusion in the radiation of single QDs and, hence, in enhancement of the degree of indistinguishability. As compared to the pure resonant pumping, implementation of the quasi-resonant one is much simpler technically, while providing reasonably high degree of indistinguishability.

Exciton relaxation process between the excited and ground states in QDs cannot be simply achieved by the interaction with acoustic phonons because of the large separation between quantized energy states. Other channels such as the Auger-type scattering and/or multiple longitudinal optical (LO) phonon scattering are required to enable the relaxation process. Recombination through the Auger-type scattering requires high carrier concentrations and, therefore, in most cases this process can be neglected [8]. Relaxation with the emission of LO phonons is possible only in those QDs in which the distances between the states are equal to the energy of one or several LO phonons. Thus, all possible channels of phonon-induced relaxation in quantum dots have significant limitations.

In this paper, we present spectroscopic studies of the excitonic spectrum and the mechanisms of exciton relaxation in InAs $/ \mathrm{Al}_{0.3} \mathrm{Ga}_{0.7} \mathrm{As}$ and $\mathrm{InAs} / \mathrm{GaAs}$ self-organized QDs. The excited excitonic states are revealed by photoluminescence (PL) spectroscopy using non-resonant over-barrier pumping [9], whereas additional information about the excited states spectrum and the mechanisms of exciton relaxation are achieved by using the quasi-resonant under-barrier selective excitation of PL (SEPL) and PL excitation (PLE) techniques [10].

\section{Experimental details}

The experiments were carried out using two series of samples fabricated by molecular beam epitaxy (MBE) [11], which contain self-organized InAs QDs grown using the Stranski-Krastanow growth mode. 
The structures were grown on a GaAs (100) substrate capped with a $200 \mathrm{~nm}$ thick GaAs buffer. The first sample (A) contains InAs QDs sandwiched between $200 \mathrm{~nm}$ thick bottom and $1.3 \mu \mathrm{m}$ thick top GaAs barrier layers. This sample can be considered as InAs QDs in the barrier $\mathrm{Al}_{x} \mathrm{Ga}_{1-x} \mathrm{As}$ when $x$ is zero. In the other sample $(\mathrm{B})$, InAs QDs are located between $200 \mathrm{~nm} \mathrm{Al}_{0.3} \mathrm{Ga}_{0.7}$ As barriers. A $1.1 \mu \mathrm{m}$ thick layer of $\mathrm{Al}_{0.4} \mathrm{Ga}_{0.6} \mathrm{As}$ alloy was grown on top of the barrier. Photonic nanoantennas in the form of columns tapered toward the apex with a diameter of $200-250 \mathrm{~nm}$ at the base and $150-200 \mathrm{~nm}$ at the top were formed from the upper $\mathrm{Al}_{0.4} \mathrm{Ga}_{0.6} \mathrm{As}$ layer by ion-beam etching with Ar ions through sapphire nanobeads [5]. The nanoantennas provide effective collection of photons into the fundamental $\mathrm{HE}_{11}$ mode and form a narrow far-field radiation pattern due to the smoothly tapered upper part [12]. As a result, collection efficiency of the QD emission can be significantly enhanced. The surface density of nanoantennas was chosen to provide optical isolation during measurements and to ensure the possibility of measuring emission spectra of a few QDs located in a single nanoantenna. In the samples of both kinds, the intended average thickness of the InAs deposition amounts to 1.7 monolayers (ML).

The PL and SEPL (PLE) spectra of the QDs were measured using a cw Ti:sapphire laser. The samples were mounted in a continuous-flow helium cryostat.

\section{Results and discussion}

The PL spectra of sample (A) under non-resonant over-barrier pumping with the excitation photon energy $1.59 \mathrm{eV}$ are shown in Fig. 1. Pump power density was varied from $3 \mathrm{~W} / \mathrm{cm}^{2}$ to $114 \mathrm{~W} / \mathrm{cm}^{2}$. At sufficiently small powers we observed one broad peak with the position of the maximum marked as $E_{0}$. This peak originates from the recombination of the ground state exciton with the subsequent emission of a photon. The peak width can be explained by the dispersion of the emission wavelengths among many involved QDs, governed by fluctuation of the QD size and shape, possible diffusion of Ga from barrier into InAs QDs and so on [13]. With increasing power density, emission peaks related to transitions involving excited states manifested themselves in the high-energy spectral range $\left(E_{1}\right.$ and $\left.E_{2}\right)$. Their emergence owes to saturation of the ground states and subsequent filling of the excited ones.

The SEPL spectra of sample (A), shown in Fig. 2, were obtained with the excitation energy varied from $1.33 \mathrm{eV}$ to $1.43 \mathrm{eV}$. The spectra are arranged with the excitation energy decreasing from top to bottom. A dashed line on the right-hand side marks the excitation energy for each spectrum. All excitation energies were below the maximum of the wetting layer (WL) emission $(1.51 \mathrm{eV})$. The excitation power was low enough to avoid any emission from an excited state and to suppress Auger scattering. When the excitation energy exceeds $1.42 \mathrm{eV}$, the spectra show a broad peak, indicating that all QDs are excited.

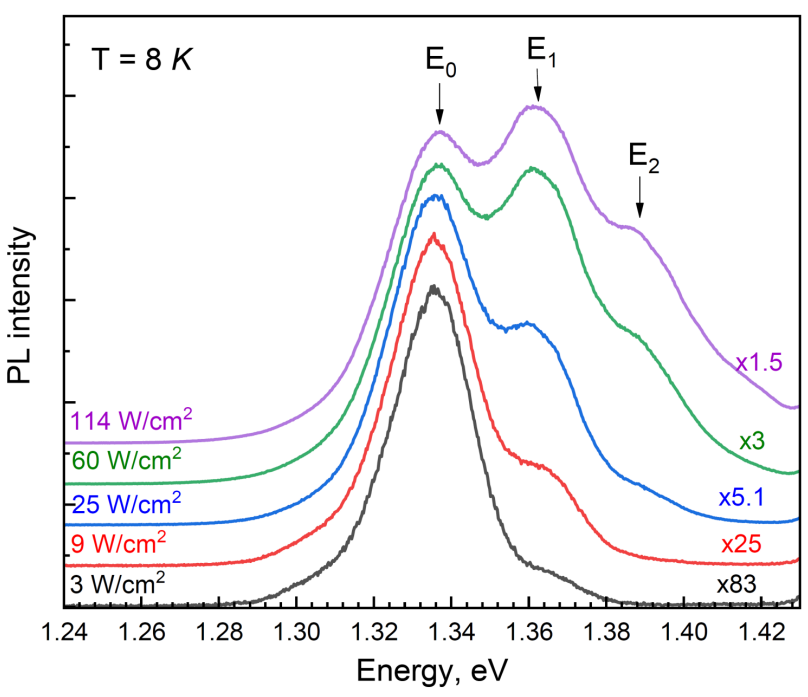

Fig. 1. PL spectra of sample (A) for different pumping powers (from $3 \mathrm{~W} / \mathrm{cm}^{2}$ to $114 \mathrm{~W} / \mathrm{cm}^{2}$ ). The spectra are normalized to the maximum intensity of the peak corresponding to ground-state exciton recombination energy $\left(E_{0}=1.336 \mathrm{eV}\right)$ and vertically offset for clarity. $E_{1}=1.362 \mathrm{eV}$ and $E_{2}=1.389 \mathrm{eV}$ denote the average positions of two first excited states.

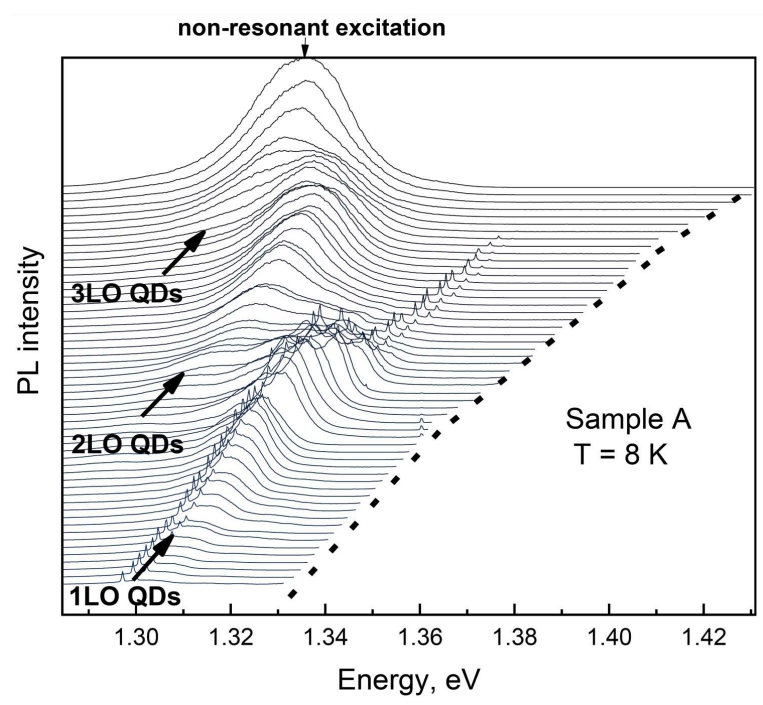

Fig. 2. SEPL spectra of sample (A) obtained at $8 \mathrm{~K}$ at different excitation energies indicated by the dash line on the right-hand side.

The position of this peak does not depend on the excitation energy, because it corresponds to non-resonant excitation through continuum tail of the WL density of states. As the excitation energy decreases, fine structures with narrower peaks are observed, owing to the selective excitation of certain QDs. The position of all these peaks strictly follow the laser excitation position at a distance corresponding exactly to particular multi-LO phonon energies, suggesting that only the QDs with their excited states located at multiple phonon energies above 
their ground state are selectively excited. Since the QDs are inhomogeneous in size, shape, elastic strain, and alloy profile, the emission energy of the resonantly excited QDs may still have a distribution. Therefore, the observed emission peak is not as narrow as that from a single QD. From the position of these peaks, we reveal different LO phonon energies corresponding to relaxation of excitons through excited states by $3 \mathrm{LO}$ QDs $(93 \mathrm{meV}), 3 \mathrm{LO} \mathrm{WL}$ (79.4 meV), 2LO QDs (65.4 meV), 2LO WL (52. meV), and 1LO QDs $(32.7 \mathrm{meV})$ phonons. Thus, we find that average LO phonon relaxation energy in QDs and WL of the sample (A) is equal to $32.7 \mathrm{meV}$ and $26.5 \mathrm{meV}$, respectively. The values are in good agreement with the data obtained in other works [14]. Additionally, two narrow lines are observed at $33.53 \mathrm{meV}$ and $36.54 \mathrm{meV}$ from excitation energy, which can be associated with the inelastic Raman scattering of the laser line with the GaAs LO and GaAs-like LO phonons in the QDs [15].

We now turn to the discussion of sample (B). Compared to sample (A), sample (B) has several significant differences. First, the use of the $\mathrm{Al}_{0.3} \mathrm{Ga}_{0.7}$ As barrier instead of GaAs made it possible to significantly increase the transition energy of the ground exciton state up to $1.77 \mathrm{eV}$. The shift in emission toward higher energy in comparison with InAs/GaAs QD arises from a combination of the large barrier band gap and from possible interdiffusion of $\mathrm{Al}$ into the quantum dots, leading to additional increase of the band gap of the QD material. Secondly, this led to a wide (up to $1.33 \mathrm{eV}$ ) spectral spreading of exciton radiation energies. Under these conditions, the study of the exciton excited states us- ing power measurements as for sample (A) is impossible. In addition, it is rather difficult to distinguish the radiation of such QDs against the background of other involved radiative transitions.

Therefore, the photonic nanoantennas were etched out of the outer $\mathrm{Al}_{0.4} \mathrm{Ga}_{0.6}$ As layer in order to isolate part of the QDs from the entire ensemble and to increase the extraction efficiency of emission from the QDs themselves. The PL spectrum of sample (B) under quasi-resonant pumping with excitation energy $1.8 \mathrm{eV}$ is shown in Fig. 3.

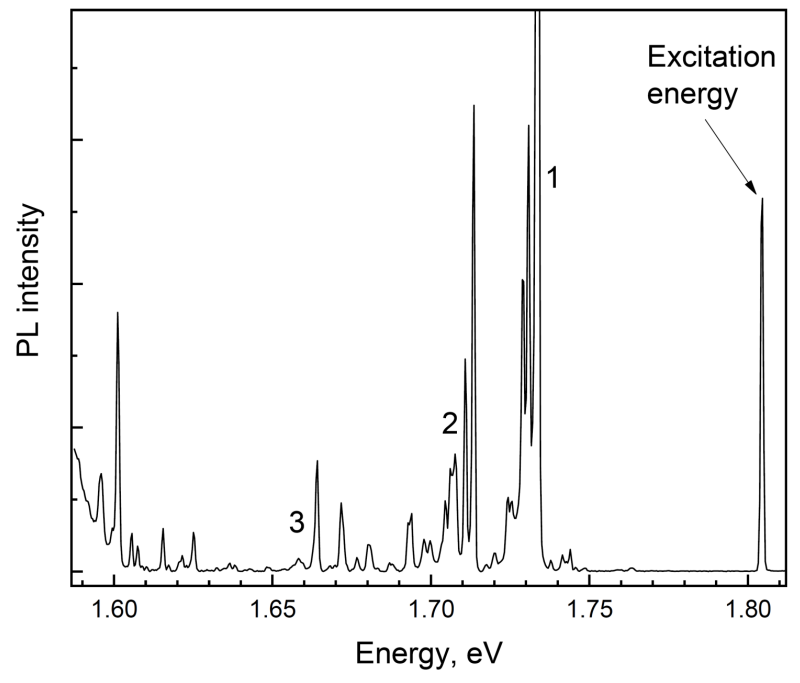

Fig. 3. PL spectrum for sample (B) obtained at $8 \mathrm{~K}$.
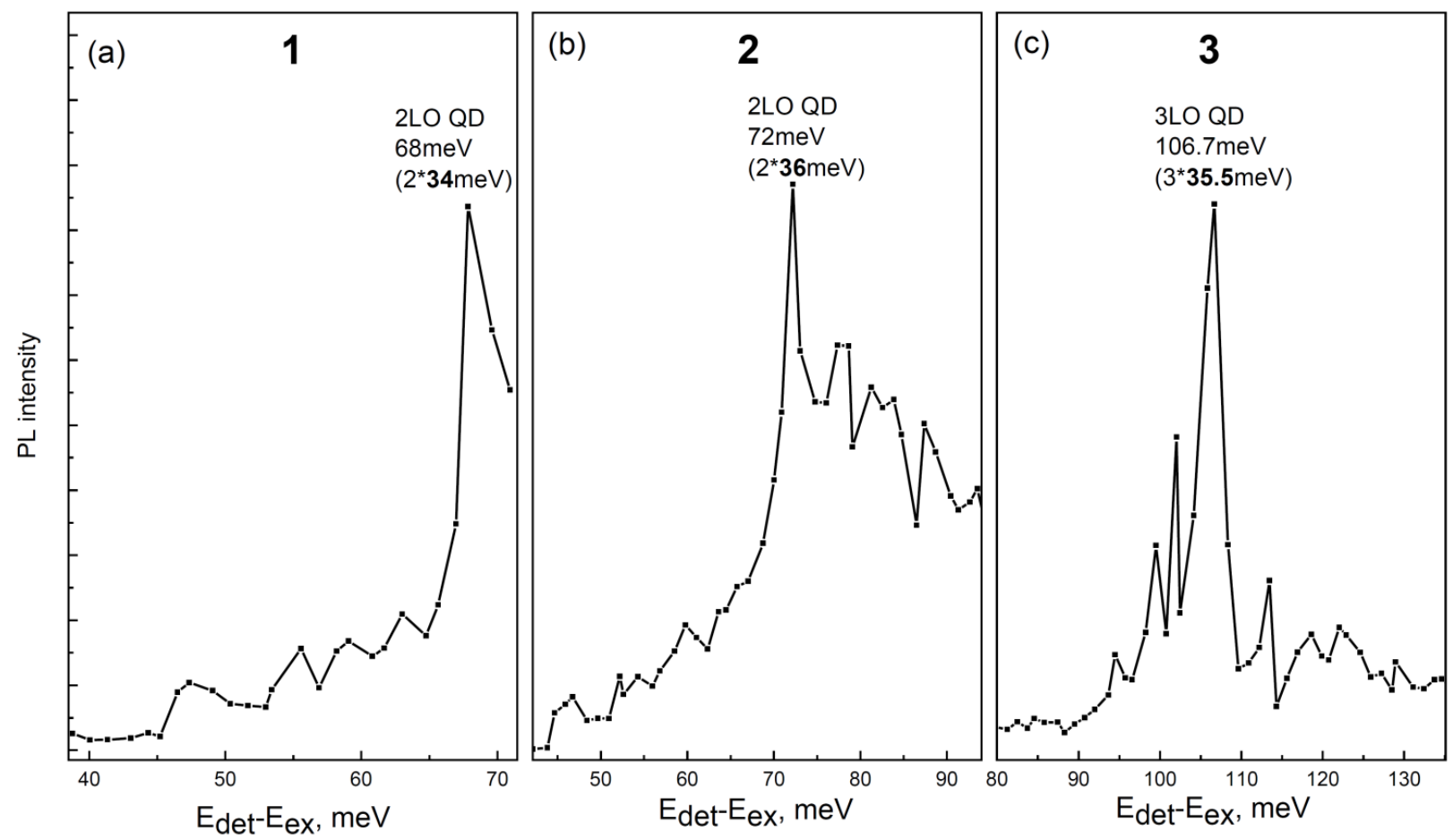

Fig. 4. PLE spectra for QDs marked as 1 (a), 2 (b) and 3 (c) in Fig. 3 (8 K). 
In this figure, we can see separate lines corresponding to the recombination of the ground state excitons in the single QDs, located within the optically excited nanoantennas. The empty gap between the excitation energy and the QDs emitting lines is related to the fact that in this spectral range there are no QDs for which the distance between the exciton excited states and the ground state would be equal to the energy of a single LO phonon. There are, however, QDs in which the exciton can relax from excited states through 2 LO phonons, 3 LO phonons, and so on.

For a more detailed study of the excited states spectrum and related relaxation mechanisms, we consider the PLE spectrum for QDs with numbers 1, 2, and 3 in Fig. 3. The PLE spectra for the QDs plotted as a function of difference between detection and excitation energies are shown in Fig. 4a-c. The excitation energy was varied from $1.72 \mathrm{eV}$ to $1.80 \mathrm{eV}$. In this figure, one can clearly see the resonance peaks, corresponding to multiphoton relaxation through excitonic excited states. We suppose that these phonons are associated with LO phonons in InAs QDs. For the first and second QDs (Fig. 4a) the resonance is observed at the distances 68 and $72 \mathrm{meV}$ which corresponds to relaxation through $2 \mathrm{LO}$ phonons with their energies 34 and $36 \mathrm{meV}$, respectively. For the third QD (Fig. 4b) the resonance is observed at $106.7 \mathrm{meV}$ that corresponds to $3 \mathrm{LO}$ phonons with their energy $35.5 \mathrm{meV}$. A higher value of the LO phonon energy as compared with the InAs QDs in the GaAs barrier can be explained by diffusion of $\mathrm{Al}$ from the AlGaAs barriers into the InAs QDs, because of which the QDs behave like an AlInAs alloy, whose LO-phonon energy is larger. The large width of the PLE resonances might be the result of partial relaxation of the $k$-selection rules.

\section{Conclusions}

We experimentally investigated excitonic spectrum in self-organized InAs $/ \mathrm{Al}_{0.3} \mathrm{Ga}_{0.7} \mathrm{As}$ QDs. For comparison, we also investigated the reference sample with zero composition of aluminum in the barrier. In the reference sample exciton excited states were revealed by PL spectroscopy using different excitation pump powers. By using selective excitation of PL we determined that an exciton relaxes from the excited states to the ground one by means of an LO multiphonon process. For the sample with $\mathrm{InAs} / \mathrm{Al}_{0.3} \mathrm{Ga}_{0.7} \mathrm{As}$ QDs, the direct determination of the excited states energies was impossible. For this reason, we carried out PLE measurements for individual QDs, located in waveguide nanoantennas. From the analysis of these measurements, we determined that excitons in this sample also relax by means of LO multiphonons, while relaxation with a single LO photon is forbidden due to, most probably, larger gap between the ground and first excited states in the InAs $/ \mathrm{Al}_{0.3} \mathrm{Ga}_{0.7} \mathrm{As}$ QDs.

\section{Acknowledgments}

This work was partially supported by the Russian Foundation for Basic Research (project no. 18-02-01212).

\section{References}

[1] M.D. Eisaman, J. Fan, A. Migdall, S.V. Polyakov, Rev. Sci. Instrum. 82, 071101 (2011).

[2] W. Zhou, J.J. Coleman, Curr. Opin. Solid State Mater. Sci. 20, 352 (2016).

[3] M.V. Rakhlin, K.G. Belyaev, G.V. Klimko, I.S. Mukhin, D.A. Kirilenko, T.V. Shubina, S.V. Ivanov, A.A. Toropov, Sci. Rep. 8, 5299 (2018).

[4] J.J. Finley, D.J. Mowbray, M.S. Skolnick, A.D. Ashmore, C. Baker, A.F.G. Monte, M. Hopkinson, Phys. Rev. B 66, 1533161 (2002).

[5] M.V. Rakhlin, K.G. Belyaev, G.V. Klimko I.V. Sedova, M.M. Kulagina, Yu.M. Zadiranov, S.I. Troshkov, Y.A. Guseva, Y.V. Terent'ev, S.V. Ivanov, A.A. Toropov, JETP Lett. 109, 145 (2019).

[6] S.M. Ulrich, S. Reitzenstein, A. Löffler, A. Forchel, P. Michler, Phys. Rev. Lett. 103, 167402 (2009).

[7] C. Santori, D. Fatal, J. Vučković, G.S. Solomon, Y. Yamamoto, Nature 419, 594 (2002).

[8] A.L. Efros, V.A. Kharchenko, M. Rosen, Solid State Commun. 93, 281 (1995).

[9] M. Grundmann, N.N. Ledentsov, O. Stier, D. Bimberg, V.M. Ustinov, P.S. Kop'ev, Zh.I. Alferov, Appl. Phys. Lett. 68, 979 (1995).

[10] H.S. Ling, C.P. Lee, M.C. Lo, J. Appl. Phys. 103, 124311 (2008).

[11] A. Polimeni, A. Patanè, M. Henini, L. Eaves, P.C. Main, Phys. Rev. B 59, 5064 (1999).

[12] I. Friedler, C. Sauvan, J. P. Hugonin, P. Lalanne, J. Claudon, J.M. Gérard, Opt. Expr. 17, 2095 (2009).

[13] A.M. Smith, S. Nie, Acc. Chem. Res. 43, 190 (2010).

[14] R. Heitz, M. Veit, N. N. Ledentsov, A. Hoffmann, D. Bimberg, V.M. Ustinov, P.S. Kop'ev, Zh.I. Alferov, Phys. Rev. B Condens. Mater. Phys. 56, 10435 (1997).

[15] S. Farfad, R. Leon, D. Leonard, J.L. Merz, P.M. Petroff, Phys. Rev. B 52, 5752 (1995). 